\title{
The GSTP1 A1578G polymorphism and the risk of childhood acute lymphoblastic leukemia: results from an updated meta-analysis
}

\author{
G.Z. Huang, W. Shan, L. Zeng and L.G. Huang \\ Department of Pediatric Surgery, West China Hospital of Sichuan University, \\ Chengdu, Sichuan Province, China \\ Corresponding author: L.G. Huang \\ E-mail: huanglugang688@163.com
}

Genet. Mol. Res. 12 (3): 2481-2491 (2013)

Received August 24, 2012

Accepted January 26, 2013

Published July 24, 2013

DOI http://dx.doi.org/10.4238/2013.July.24.3

\begin{abstract}
Studies investigating the association between the glutathione S-transferase P1 (GSTP1) A1578G polymorphism and the risk of childhood acute lymphoblastic leukemia (ALL) report conflicting results. The aim of this study was to quantitatively summarize the evidence for such a relationship. Two investigators independently searched the Medline, Embase, China National Knowledge Infrastructure, and Wangfang databases for studies of the polymorphism and ALL. Summary odds ratios (ORs) and 95\% confidence intervals (CIs) for the GSTP1 polymorphism and childhood ALL were calculated in a fixed-effect model. Pooled ORs were calculated for a co-dominant model (GG vs AA, AG vs AA), a dominant model (GG + AG vs AA), and a recessive model $(\mathrm{GG} v s \mathrm{AA}+\mathrm{AG})$. Analyses were also performed in subgroups stratified by race, study design, genotyping methods, and study sample size. This meta-analysis included 8 case-control studies with 1384 childhood ALL cases and 1755 controls. Overall, the variant genotypes (GG and AG) of A1578G were not associated with childhood
\end{abstract}


ALL risk, when compared with the wild-type homozygote AA genotype $(\mathrm{GG} v s \mathrm{AA}, \mathrm{OR}=1.09,95 \% \mathrm{CI}=0.84-1.43 ; \mathrm{AG} v s \mathrm{AA}, \mathrm{OR}=1.05$, $95 \% \mathrm{CI}=0.91-1.23)$. Similarly, no associations were found in the dominant and recessive models (dominant model, $\mathrm{OR}=1.06,95 \% \mathrm{CI}=$ 0.92-1.23; recessive model, $\mathrm{OR}=1.09,95 \% \mathrm{CI}=0.84-1.43$ ). Stratified analyses did not detect significant association in any subgroup. No heterogeneity or publication bias was observed in the present study. This updated meta-analysis indicates that the GSTP1 A1578G polymorphism is not associated with the risk of childhood ALL. In the future, additional studies in Asian and African-American patients should be performed to re-evaluate the association in these populations.

Key words: Acute lymphoblastic leukemia; Childhood; Meta-analysis; GSTP1 polymorphism;

\section{INTRODUCTION}

Acute lymphoblastic leukemia (ALL) is the most common pediatric cancer and accounts for approximately $25-30 \%$ of all childhood malignancies. The occurrence of pediatric leukemia has been linked to several environmental, maternal, and paternal factors and to the exposure to various biological, physical, and chemical factors (Whyatt and Perera, 1995; Severson and Ross, 1999). Despite much investigation, the causes of ALL are not yet fully understood. Like many other cancers, acute leukemia is considered to be a complex disease caused by a combination of genetic and environmental factors (Arruda et al., 2001; Krajinovic et al., 2001). Children are particularly vulnerable to environmental toxins because of their greater relative exposure, immature metabolism, and higher levels of cell division and growth (Perera, 1997; Krajinovic et al., 2002a). Polymorphisms in genes encoding xenobiotic-metabolizing enzymes are largely responsible for inter-individual differences in the ability to activate and detoxify mutagenic/carcinogenic agents, and therefore may influence the susceptibility to cancer (Idle, 1991; Nebert, 1991).

Glutathione $S$-transferases (GSTs) are major phase II detoxifying enzymes that catalyze the conjugation of activated xenobiotics to an endogenous water soluble substrate, such as glutathione, uridine diphosphate glucuronic acid, or glycine (Millar et al., 1999). The GSTs are part of a complex and widespread enzyme superfamily that has been subdivided into 8 classes (Strange et al., 2001). Differences in the activities of some GSTs are determined by genetic polymorphisms. GSTP1 encodes the enzyme glutathione S-transferase P1 and is located on chromosome 11q13. Polymorphisms in GSTP1 were first reported by Board et al. (1989). They include an A $\rightarrow \mathrm{G}$ transition at nucleotide 313 in exon $5(G S T P 1 * B)$ and a $\mathrm{G} \rightarrow \mathrm{T}$ transversion at nucleotide 341 in exon 6 $\left(G S T P 1{ }^{*} C\right)$, resulting in the substitution of Ile $\rightarrow \mathrm{Val}$ and $\mathrm{Val} \rightarrow \mathrm{Ala}$, respectively, in the active site of the enzyme. These allele variants appear to reduce GSTP1 activity. A decrease in GST enzyme activity could result in the inefficient detoxification of various carcinogens, which could in turn lead to genetic damage and increase cancer risk (Harries et al., 1997; Ryberg et al., 1997).

Over the last 2 decades, a number of case-control studies have been conducted to investigate the association between the GSTP1 A1578G polymorphism and the risk of childhood ALL. However, the results of these studies are conflicting. Recently, Vijayakrishnan and Houlston (2010) published a meta-analysis to assess the association between candidate gene polymorphisms 
and the risk of childhood ALL and found that the GSTP1 A1578G polymorphism was not associated with the risk of childhood ALL. However, Vijayakrishnan and Houlston's study had some limitations such as relatively small sample size, failure to include an important study, and some of the data (revealed in Vijayakrishnan and Houlston's Table S2, 2010), to our knowledge, were incorrect. In order to obtain a more comprehensive estimate of the association between the GSTP1 A1578G polymorphism and the risk of childhood ALL, we conducted an updated meta-analysis to re-evaluate this association.

\section{MATERIAL AND METHODS}

\section{Publication search}

We searched the PubMed, Embase, China National Knowledge Infrastructure, and Wangfang databases for all articles on the association between GSTP1 polymorphisms and childhood ALL risk (last search update August 15, 2012). The following key words were used: "acute lymphoblastic leukaemia" or "ALL," "GSTP1" or "glutathione s-transferase P1," and "polymorphism" or "variant". The search was not restricted by language and conducted on human subject studies. Simultaneously, the reference lists of previous reviews and meta-analyses were searched manually. If more than one article was published by the same author using the same case series, we selected the study where the most number of individuals were investigated.

\section{Inclusion and exclusion criteria}

We reviewed the abstracts of all citations and retrieved studies. The following criteria were used to include published studies: i) case-control studies were conducted to evaluate the association between the GSTP1 A1578G polymorphism and the risk of childhood ALL; ii) sufficient genotype data were presented to calculate the odds ratios (ORs) and 95\% confidence intervals (CIs); and iii) the paper clearly described the sources of cases and controls. Major reasons for the exclusion of studies were i) no control, ii) duplicated studies, and iii) sufficient data were not reported.

\section{Data extraction}

Two investigators (HG and SW) extracted information from all eligible publications independently according to the inclusion criteria listed above. Disagreements were resolved by discussion between the two investigators. The following characteristics were collected from each study: first author, year of publication, country of the first or corresponding author, ethnicity, number of cases and controls, study design [population-based case-control (PCC), hospital-based case-control (HCC)], genotyping methods, minor allele frequency in controls, and evidence of Hardy-Weinberg equilibrium (HWE) (Table 1).

\section{Statistical analysis}

We first assessed HWE in the controls for each study using the goodness-of-fit test (chi-square or Fisher exact test) and $\mathrm{P}<0.05$ was considered as significant disequilibrium. The strength of the association between childhood ALL and the GSTP1 A1578G polymor- 
phism was estimated using ORs, with the corresponding 95\%CIs. Pooled ORs were calculated under a co-dominant model (GG vs AA, AG vs AA), a dominant model (GG $+\mathrm{AG}$ vs AA), and a recessive model (GG vs AG + AA). We also performed subgroup analyses by ethnicity, study sample size ( $>500 / \leq 500$ subjects), genotyping methods, and source of controls (HCC/PCC).

Both the Cochran's Q statistic (Cochran, 1954) to test for heterogeneity and the $I^{2}$ statistic to quantify the proportion of the total variation due to heterogeneity (Higgins et al., 2003) were calculated. A P value greater than the nominal level of 0.10 for the $Q$ statistic indicated a lack of heterogeneity across studies, allowing for the use of a fixed-effect model (the Mantel-Haenszel method) (Mantel and Haenszel, 1959); otherwise, the random-effect model (the DerSimonian and Laird method) was used (DerSimonian and Laird, 1986). Sensitivity analysis was performed to assess the stability of the results.

Several methods were used to assess potential publication bias. Visual inspection of funnel plot asymmetry was conducted. The Begg's rank correlation method (Begg and Mazumdar, 1994) and the Egger's weighted regression method (Egger et al., 1997) were used to statistically assess publication bias ( $\mathrm{P}<0.05$ was considered to be statistically significant). All analyses were done using the STATA software, version 11.0 (StataCorp, USA). All the P values were two-sided.

\section{RESULTS}

\section{Characteristics of the studies}

We identified 38 relevant studies by searching the databases. Ten publications described the association between the GSTP1 polymorphism and childhood ALL. Their full texts were retrieved and carefully studied. Finally, a total of 8 eligible studies involving 1384 cases and 1755 controls were included in the pooled analyses (Krajinovic et al., 2002b; Balta et al., 2003; Barnette et al., 2004; Canalle et al., 2004; Clavel et al., 2005; Gatedee et al., 2007; Pigullo et al., 2007; Chan et al., 2011). The characteristics of the selected studies are summarized in Table 1. Ten studies were performed in Caucasian patients and 2 studies were performed in Asian patients. The studies were carried out in Canada, Turkey, USA, Brazil, France, Thailand, Italy, and Indonesia. Controls were mainly healthy children and were matched by age and/or gender. Six studies selected controls in a population-based manner whereas 2 used hospital-based controls. All studies extracted DNA from peripheral blood and a classic polymerase chain reaction-restriction fragment length polymorphism (PCR-RFLP) assay was used in 5 of 8 studies. The distribution of genotypes in the controls of all studies was in agreement with HWE.

\section{Quantitative synthesis}

Table 2 lists the main results of this meta-analysis and Figure 1A-D show the association of childhood ALL risk with the GSTP1 A1578G polymorphism. Overall, the variant genotypes (GG and AG) of the polymorphism were not associated with childhood ALL risk when compared with the wild-type AA homozygote ( $\mathrm{GG} v s \mathrm{AA}, \mathrm{OR}=1.09,95 \% \mathrm{CI}=0.84-1.43$; $\mathrm{AG}$ vs $\mathrm{AA}, \mathrm{OR}=1.05,95 \% \mathrm{CI}=0.91-1.23$ ), without any between-study heterogeneity. Similarly, no associations were observed in the dominant or recessive models (dominant model, OR = $1.06,95 \% \mathrm{CI}=0.92-1.23$; recessive model, $\mathrm{OR}=1.09,95 \% \mathrm{CI}=0.84-1.43$ ). 
GSTP1 polymorphism and childhood ALL risk

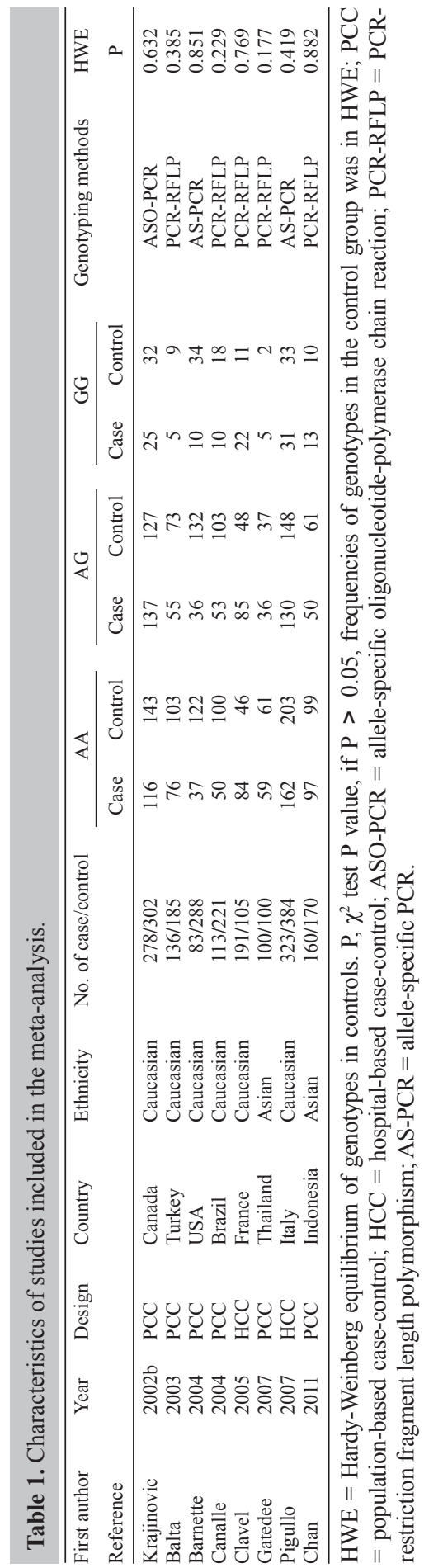


Table 2. Stratified analyses of the GSTP1 A1578G polymorphism and risk of childhood acute lymphoblastic leukemia.

\begin{tabular}{|c|c|c|c|c|c|c|c|c|c|}
\hline \multirow[t]{2}{*}{ Variables } & \multirow[t]{2}{*}{$\mathrm{N}$} & \multicolumn{2}{|l|}{ GG $v s \mathrm{AA}$} & \multicolumn{2}{|l|}{$\mathrm{AG} v s \mathrm{AA}$} & \multicolumn{2}{|c|}{ Dominant model } & \multicolumn{2}{|c|}{ Recessive model } \\
\hline & & OR $(95 \% \mathrm{CI})$ & $\mathrm{P}$ & OR $(95 \% \mathrm{CI})$ & $\mathrm{P}$ & OR $(95 \% \mathrm{CI})$ & $\mathrm{P}$ & OR $(95 \% \mathrm{CI})$ & $\mathrm{P}$ \\
\hline Total & 8 & $1.09(0.84-1.43)$ & 0.96 & $1.05(0.91-1.23)$ & 0.86 & $1.06(0.92-1.23)$ & 0.95 & $1.09(0.84-1.43)$ & 0.96 \\
\hline \multicolumn{10}{|l|}{ Ethnicity } \\
\hline Asian & 2 & $1.54(0.72-3.32)$ & 0.49 & $0.90(0.62-1.29)$ & 0.63 & $0.97(0.69-1.37)$ & 0.62 & $1.54(0.72-3.32)$ & 0.49 \\
\hline Caucasian & 6 & $1.04(0.78-1.39)$ & 0.99 & $1.09(0.92-1.29)$ & 0.83 & $1.08(0.92-1.27)$ & 0.90 & $1.04(0.78-1.39)$ & 0.99 \\
\hline \multicolumn{10}{|l|}{ Study design } \\
\hline $\mathrm{HCC}$ & 2 & $1.15(0.74-1.80)$ & 0.88 & $1.06(0.81-1.39)$ & 0.68 & $1.08(0.84-1.39)$ & 0.69 & $1.15(0.74-1.80)$ & 0.88 \\
\hline PCC & 6 & $1.06(0.76-1.49)$ & 0.87 & $1.05(0.87-1.27)$ & 0.68 & $1.05(0.88-1.26)$ & 0.85 & $1.06(0.76-1.49)$ & 0.86 \\
\hline \multicolumn{10}{|c|}{ Genotyping methods } \\
\hline PCR-RFLP & 5 & $1.16(0.76-1.78)$ & 0.82 & $0.97(0.78-1.20)$ & 0.97 & $0.99(0.81-1.23)$ & 0.99 & $1.16(0.76-1.78)$ & 0.82 \\
\hline Other & 3 & $1.05(0.74-1.49)$ & 0.86 & $1.14(0.93-1.41)$ & 0.45 & $1.13(0.92-1.38)$ & 0.57 & $1.05(0.74-1.49)$ & 0.86 \\
\hline \multicolumn{10}{|l|}{ Study sample s } \\
\hline$>500$ & 2 & $1.07(0.73-1.59)$ & 0.62 & $1.20(0.95-1.51)$ & 0.43 & $1.18(0.94-1.47)$ & 0.60 & $1.07(0.73-1.59)$ & 0.62 \\
\hline$\leq 500$ & 6 & $1.11(0.77-1.62)$ & 0.89 & $0.96(0.78-1.17)$ & 0.99 & $0.98(0.81-1.19)$ & 0.99 & $1.11(0.77-1.62)$ & 0.89 \\
\hline
\end{tabular}

$\mathrm{N}=$ number of comparisons; P value of the Q-test for heterogeneity test. Random-effect model was used when $\mathrm{P}$ value for heterogeneity test $<0.1$; otherwise, fixed-effect model was used. $\mathrm{HCC}=$ hospital-based case-control studies; $\mathrm{PCC}=$ population-based case-control studies.

On the basis of the potential underestimation of the true effect of the polymorphism on ALL risk, we stratified these studies according to ethnicity, source of controls, genotyping methods, and study sample size. Different ethnicities were categorized as Asian and Caucasian. Different genotyping methods were defined as PCR-RFLP and other. In the stratified analyses, the variant genotypes (GG and $A G)$ had no significant relationship with childhood ALL in all of the subgroups compared with the wild-type AA. Similar results were observed in the recessive and dominant models (Table 2).

\section{Heterogeneity and sensitivity analyses}

Significant heterogeneity between studies was not observed in overall comparisons and subgroup analyses. In the sensitivity analysis, the influence of each study on the pooled OR was examined by repeating the meta-analysis while omitting each study, one at a time. This procedure confirmed the stability of our overall results.

\section{Publication bias}

Funnel plot, Begg and Egger tests were used to evaluate publication bias of the literature on childhood ALL. Figure 2 displays the funnel plot that examines the GSTP1 A1578G polymorphism and overall childhood ALL risk included in the meta-analysis in the homozygous comparison. The shape of funnel plots did not reveal any evidence of funnel plot asymmetry. The statistical results did not show publication bias either (GG vs AA: Begg test, $\mathrm{P}=0.39$, Egger test, $\mathrm{P}=0.41$; AG vs $\mathrm{AA}$ : Begg test, $\mathrm{P}=0.17$, Egger test, $\mathrm{P}=0.06$; dominant model: Begg test, $\mathrm{P}=0.71$, Egger test, $\mathrm{P}=0.08$; recessive model: Begg test, $\mathrm{P}=0.39$, Egger test, $\mathrm{P}=0.41$ ). 


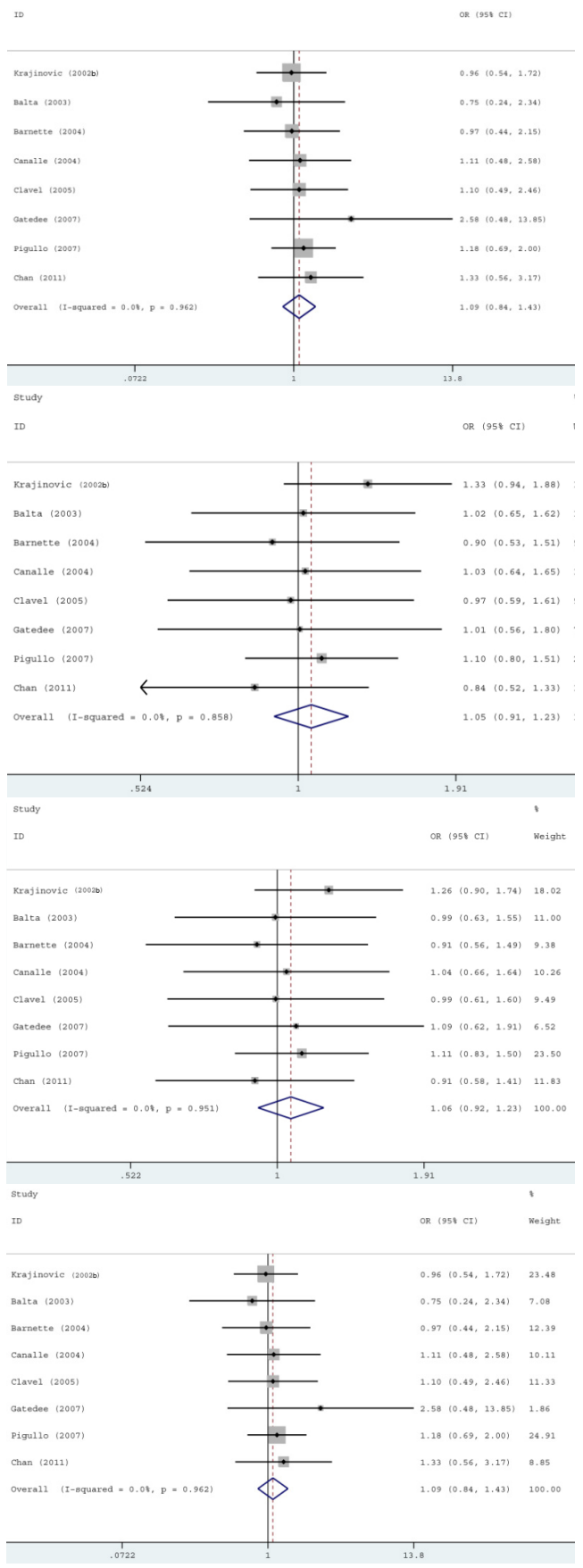

Figure 1. Forest plots of odds ratio (ORs) with $95 \%$ confidence intervals (CIs) for the GSTP1 A1578G polymorphism and risk of childhood acute lymphoblastic leukemia. The center of each square represents the OR, the area of the square is the number of sample and thus the weight used in the meta-analysis, and the horizontal line indicates the 95\%CI. A. GG vs AA. B. AG vs AA. C. GG + AG vs AA. D. GG vs AG + AA. 


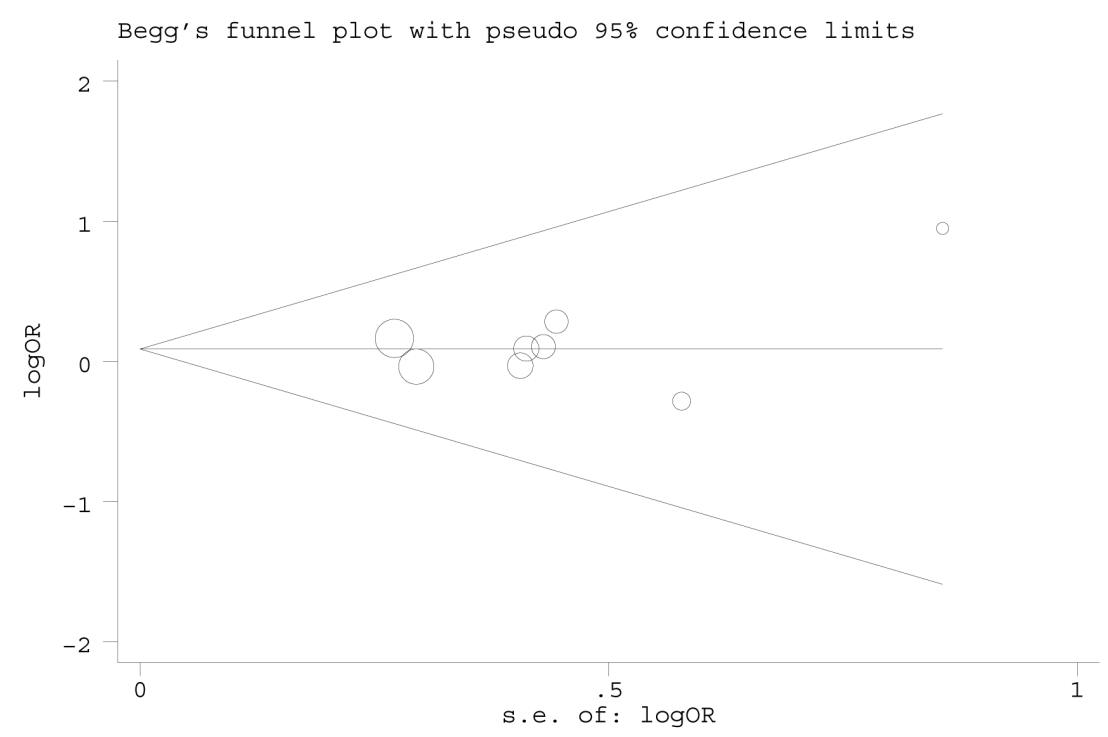

Figure 2. Funnel plot for publication bias test (GG vs AA). Each point represents a separate study for the indicated association.

\section{DISCUSSION}

ALL is the most frequent malignancy affecting children, accounting for $30 \%$ of all childhood cancers. Despite much investigation, the causes of this disease are not yet fully understood. Like many other cancers, ALL is considered to be a complex disease caused by a combination of genetic and environmental factors (Arruda et al., 2001; Krajinovic et al., 2001). In this context, understanding the interactions between the various predisposing genes and environmental factors in the pathogenesis of childhood leukemia is of considerable public health importance. Biological markers of individual susceptibility could prove useful for identifying persons at risk for developing leukemia and for targeting preventive strategies. The GSTs are a family of enzymes involved in the detoxification of a wide range of chemicals, including important environmental carcinogens [e.g., benzo(a)pyrene and other polycyclic aromatic hydrocarbons] (Perera, 1996; Hengstler et al., 1998). Human GSTP1 has been shown to catalyze the isomerization of 13-cisretioic acid to all-transretioic acid (Fukai et al., 1992). As a result, GSTP1 is commonly studied for its effect on the susceptibility to acute myeloid leukemia (Allan et al., 2001). In recent years, a number of molecular epidemiologic studies have been conducted to evaluate the role of the A1578G polymorphism in the GSTP1 gene on childhood ALL risk; however, the results remain conflicting rather than conclusive (Krajinovic et al., 2002b; Balta et al., 2003; Barnette et al., 2004; Canalle et al., 2004; Clavel et al., 2005; Gatedee et al., 2007; Pigullo et al., 2007; Chan et al., 2011). Meta-analysis is a powerful statistical method that can provide a quantitative approach for pooling the results of different studies on the same topic, and thus a systematic review and meta-analysis of the association between the GSTP1 A1578G polymorphism and childhood ALL risk is of great value.

This updated analysis, based on 8 case-control studies with 1384 cases and 1755 controls explored the association between the GSTP1 A1578G polymorphism and the risk of 
childhood ALL. Consistent with the previous meta-analysis by Vijayakrishnan and Houlston (2010), our findings indicate that the GSTP1 A1578G polymorphism is not associated with childhood ALL risk. Even after stratifying the studies by ethnicity, study sample size, genotyping methods, and source of controls, the results were similar. Moreover, our results are consistent with previous meta-analyses based on other cancers. For example, 3 previous metaanalyses confirmed that the GSTP1 A1578G polymorphism is not associated with the risk for thyroid cancer ( $\mathrm{Li}$ et al., 2012), colorectal cancer (Gao et al., 2009), or ovarian cancer (Economopoulos et al., 2010). However, Lu et al. (2011) concluded that the GSTP1 A1578G polymorphism may increase the susceptibility to breast cancer in an Asian population. Similarly, Kellen et al. (2007) found that the GSTP1 A1578G polymorphism was associated with a modest increase in the risk for bladder cancer, and Zhou et al. (2009) found that the GSTP1 A1578G polymorphism may be associated with gastric cancer in Caucasians. Although the reasons for these apparent differences in risk for different tumors are as yet unknown, some possibilities should be considered. First, these genetic associations may vary in different types of cancer due to the different mechanisms of carcinogenesis in each cancer. Second, the use of different ethnic populations in the studies may contribute to the discrepancy. The inclusion of different studies performed in different populations may cause variability among meta-analyses results. Third, some methodological diversity, such as differences in inclusion criteria, the quality of the original studies included, selection bias, type I error, and small sample size may also contribute to the discrepancy.

Results of meta-analyses often depend on the procedures used for selecting the control population (Benhamou et al., 2002). Different sources of controls may be a confounding factor that may impact the conclusion of our study. For instance, some included studies used healthy children from the general population as the reference group, whereas others selected hospitalized children without ALL as the reference group. In order to eliminate interference from this confounding factor, we performed a subgroup analysis by stratifying studies based on the source of controls. Our results showed that there was no significant association between the GSTP1 A1578G polymorphism and childhood ALL risk upon including only controls collected in hospitals or those collected only in the population, thus confirming the reliability of our overall results.

One of the major concerns in performing a sound meta-analysis is the degree of heterogeneity that exists between the component studies because non-homogeneous data are liable to result in misleading results. In the present study, the Q-test and $I^{2}$ statistics were used to test the significance of heterogeneity. Obvious heterogeneity between studies was not observed in overall comparisons and subgroup analyses. Another important issue for any metaanalysis is publication bias due to the selective publication of reports. In the current study, Funnel plot, Begg and Egger tests were performed to evaluate this problem. Both the shape of funnel plots and statistical results did not show publication bias.

However, there still exist some limitations in this meta-analysis: 1) only published studies were included in the meta-analysis; therefore, publication bias may have occurred, even though the use of a statistical test did not reveal it; 2) our meta-analysis was based on unadjusted OR estimates because not all published studies presented adjusted ORs or when they did, the ORs were not adjusted by the same potential confounders, such as age, gender, ethnicity, and exposures; the lack of this information may cause serious bias; 3) meta-analysis is a retrospective approach that is subject to methodological limitations. In order to minimize bias, we developed a detailed protocol before initiating the study, and performed a meticulous 
search for published studies using explicit methods for study selection, data extraction, and data analysis. Nevertheless, our results should still be interpreted with caution.

In conclusion, this updated meta-analysis indicates that the GSTP1 A1578G polymorphism is not associated with altered susceptibility to childhood ALL. Since few studies were performed in non-Caucasian population, it is critical that larger and welldesigned multicentric studies in Asian and African-American patients should be performed to re-evaluate this association.

\section{REFERENCES}

Allan JM, Wild CP, Rollinson S, Willett EV, et al. (2001). Polymorphism in glutathione S-transferase P1 is associated with susceptibility to chemotherapy-induced leukemia. Proc. Natl. Acad. Sci. U. S. A. 98: 11592-11597.

Arruda VR, Lima CS, Grignoli CR, de Melo MB, et al. (2001). Increased risk for acute myeloid leukaemia in individuals with glutathione S-transferase mu 1 (GSTM1) and theta 1 (GSTT1) gene defects. Eur. J. Haematol. 66: 383-388.

Balta G, Yuksek N, Ozyurek E, Ertem U, et al. (2003). Characterization of MTHFR, GSTM1, GSTT1, GSTP1, and CYP1A1 genotypes in childhood acute leukemia. Am. J. Hematol. 73: 154-160.

Barnette P, Scholl R, Blandford M, Ballard L, et al. (2004). High-throughput detection of glutathione s-transferase polymorphic alleles in a pediatric cancer population. Cancer Epidemiol. Biomarkers Prev. 13: 304-313.

Begg CB and Mazumdar M (1994). Operating characteristics of a rank correlation test for publication bias. Biometrics 50: 1088-1101.

Benhamou S, Lee WJ, Alexandrie AK, Boffetta P, et al. (2002). Meta- and pooled analyses of the effects of glutathione S-transferase M1 polymorphisms and smoking on lung cancer risk. Carcinogenesis 23: 1343-1350.

Board PG, Webb GC and Coggan M (1989). Isolation of a cDNA clone and localization of the human glutathione S-transferase 3 genes to chromosome bands 11q13 and 12q13-14. Ann. Hum. Genet. 53: 205-213.

Canalle R, Burim RV, Tone LG and Takahashi CS (2004). Genetic polymorphisms and susceptibility to childhood acute lymphoblastic leukemia. Environ. Mol. Mutagen. 43: 100-109.

Chan JY, Ugrasena DG, Lum DW, Lu Y, et al. (2011). Xenobiotic and folate pathway gene polymorphisms and risk of childhood acute lymphoblastic leukaemia in Javanese children. Hematol. Oncol. 29: 116-123.

Clavel J, Bellec S, Rebouissou S, Menegaux F, et al. (2005). Childhood leukaemia, polymorphisms of metabolism enzyme genes, and interactions with maternal tobacco, coffee and alcohol consumption during pregnancy. Eur. J. Cancer Prev. 14: 531-540.

Cochran WG (1954). The combination of estimates from different experiments. Biometrics 10: 101-129.

DerSimonian R and Laird N (1986). Meta-analysis in clinical trials. Control Clin. Trials 7: 177-188.

Economopoulos KP, Sergentanis TN and Vlahos NF (2010). Glutathione S-transferase M1, T1, and P1 polymorphisms and ovarian cancer risk: a meta-analysis. Int. J. Gynecol. Cancer 20: 732-737.

Egger M, Davey SG, Schneider M and Minder C (1997). Bias in meta-analysis detected by a simple, graphical test. $B M J$ 315: 629-634.

Fukai F, Ohtaki H, Ueda T and Katayama T (1992). A possible role of glutathione S-transferase in rat ovary and testes. $J$. Clin. Biochem. Nutr. 12: 93-107.

Gao Y, Pan X, Su T, Mo Z, et al. (2009). Glutathione S-transferase P1 Ile105Val polymorphism and colorectal cancer risk: a meta-analysis and HuGE review. Eur. J. Cancer 45: 3303-3314.

Gatedee J, Pakakassama S, Muangman S and Pongstaporn W (2007). Glutathione S-transferase P1 genotypes, genetic susceptibility and outcome of therapy in thai childhood acute lymphoblastic leukemia. Asian Pac. J. Cancer Prev. 8: 294-296.

Harries LW, Stubbins MJ, Forman D, Howard GC, et al. (1997). Identification of genetic polymorphisms at the glutathione S-transferase Pi locus and association with susceptibility to bladder, testicular and prostate cancer. Carcinogenesis 18: 641-644.

Hengstler JG, Arand M, Herrero ME and Oesch F (1998). Polymorphisms of N-acetyltransferases, glutathione S-transferases, microsomal epoxide hydrolase and sulfotransferases: influence on cancer susceptibility. Recent Results Cancer Res. 154: 47-85.

Higgins JP, Thompson SG, Deeks JJ and Altman DG (2003). Measuring inconsistency in meta-analyses. BMJ 327: 557-560. Idle JR (1991). Is environmental carcinogenesis modulated by host polymorphism? Mutat. Res. 247: 259-266.

Kellen E, Hemelt M, Broberg K, Golka K, et al. (2007). Pooled analysis and meta-analysis of the glutathione S-transferase P1 Ile 105Val polymorphism and bladder cancer: a HuGE-GSEC review. Am. J. Epidemiol. 165: 1221-1230. 
Krajinovic M, Labuda D and Sinnett D (2001). Childhood acute lymphoblastic leukemia: genetic determinants of susceptibility and disease outcome. Rev. Environ. Health 16: 263-279.

Krajinovic M, Sinnett H, Richer C, Labuda D, et al. (2002a). Role of NQO1, MPO and CYP2E1 genetic polymorphisms in the susceptibility to childhood acute lymphoblastic leukemia. Int. J. Cancer 97: 230-236.

Krajinovic M, Labuda D and Sinnett D (2002b). Glutathione S-transferase P1 genetic polymorphisms and susceptibility to childhood acute lymphoblastic leukaemia. Pharmacogenetics 12: 655-658.

Li J, Long J, Hu Y, Tan A, et al. (2012). Glutathione S-transferase M1, T1, and P1 polymorphisms and thyroid cancer risk: a meta-analysis. Cancer Epidemiol. 36: e333-e340.

Lu S, Wang Z, Cui D, Liu H, et al. (2011). Glutathione S-transferase P1 Ile105Val polymorphism and breast cancer risk: a meta-analysis involving 34,658 subjects. Breast Cancer Res. Treat. 125: 253-259.

Mantel N and Haenszel W (1959). Statistical aspects of the analysis of data from retrospective studies of disease. J. Natl. Cancer Inst. 22: 719-748.

Millar DS, Ow KK, Paul CL, Russell PJ, et al. (1999). Detailed methylation analysis of the glutathione S-transferase pi (GSTP1) gene in prostate cancer. Oncogene 18: 1313-1324.

Nebert DW (1991). Role of genetics and drug metabolism in human cancer risk. Mutat. Res. 247: 267-281.

Perera FP (1996). Molecular epidemiology: insights into cancer susceptibility, risk assessment, and prevention. J. Natl. Cancer Inst. 88: 496-509.

Perera FP (1997). Environment and cancer: who are susceptible? Science 278: 1068-1073.

Pigullo S, Haupt R, Dufour C, Di MP, et al. (2007). Are genotypes of glutathione S-transferase superfamily a risk factor for childhood acute lymphoblastic leukemia? Results of an Italian case-control study. Leukemia 21: 1122-1124.

Ryberg D, Skaug V, Hewer A, Phillips DH, et al. (1997). Genotypes of glutathione transferase M1 and P1 and their significance for lung DNA adduct levels and cancer risk. Carcinogenesis 18: 1285-1289.

Severson RK and Ross JA (1999). The causes of acute leukemia. Curr. Opin. Oncol. 11: 20-24.

Strange RC, Spiteri MA, Ramachandran S and Fryer AA (2001). Glutathione-S-transferase family of enzymes. Mutat. Res. 482: 21-26.

Vijayakrishnan J and Houlston RS (2010). Candidate gene association studies and risk of childhood acute lymphoblastic leukemia: a systematic review and meta-analysis. Haematologica 95: 1405-1414.

Whyatt RM and Perera FP (1995). Application of biologic markers to studies of environmental risks in children and the developing fetus. Environ. Health Perspect. 103: 105-110.

Zhou Y, Li N, Zhuang W, Yin YQ, et al. (2009). Glutathione S-transferase P1 gene polymorphism associated with gastric cancer among Caucasians. Eur. J. Cancer 45: 1438-1442. 